\title{
QTL Mapping of Adult-Plant Resistance to Stripe Rust in Chinese Wheat Cultivar Chuanyu 16
}

\author{
Ling $\mathrm{Wu}$
}

Institute of Crop Science, National Wheat Improvement Center

The National Key Facility for Crop Gene Resources and Genetic Improvement

Chinese Academy of Agricultural Sciences (CAAS), 12 Zhongguancun South Street, Beijing 100081, China

Triticeae Research Institute, Sichuan Agricultural University, Chengdu 611130, Sichuan, China

Crop Research Institute, Sichuan Academy of Agricultural Science, Chengdu 610066, Sichuan, China

Key Laboratory of Biology and Genetic Breeding in Wheat (Southwest), Ministry of Agriculture, China

$$
\text { Youliang Zheng }
$$

Triticeae Research Institute, Sichuan Agricultural University, Chengdu 611130, Sichuan, China

\section{Xianchun Xia}

Institute of Crop Science, National Wheat Improvement Center

The National Key Facility for Crop Gene Resources and Genetic Improvement

Chinese Academy of Agricultural Sciences (CAAS), 12 Zhongguancun South Street, Beijing 100081, China

\section{Yunliang Peng}

Plant Protection Institute, Sichuan Academy of Agricultural Science, Chengdu 610066, Sichuan, China

\section{Huazhong Zhu}

Crop Research Institute, Sichuan Academy of Agricultural Science, Chengdu 610066, Sichuan, China

$$
\text { Yongjian Liu }
$$

College of Agriculture, Sichuan Agricultural University, Chengdu 611130, Sichuan, China

$\mathrm{Yu} \mathrm{Wu}$

Chengdu Institute of Biology, Chinese Academy of Science, Chengdu 610041, Sichuan, China

\section{Shizhao Li}

Crop Research Institute, Sichuan Academy of Agricultural Science, Chengdu 610066, Sichuan, China

Zhonghu He (Corresponding author)

Institute of Crop Science, National Wheat Improvement Center

The National Key Facility for Crop Gene Resources and Genetic Improvement

Chinese Academy of Agricultural Sciences (CAAS), 12 Zhongguancun South Street, Beijing 100081, China

International Maize and Wheat Improvement Center (CIMMYT) China Office, c/o CAAS

12 Zhongguancun South Street, Beijing 100081, China

E-mail: zhhecaas@163.com 
Received: July 11, 2011

doi:10.5539/jas.v4n3p57
Accepted: August 2, $2011 \quad$ Online Published: December 29, 2011

URL: http://dx.doi.org/10.5539/jas.v4n3p57

\begin{abstract}
Stripe rust, caused by Puccinia striiformis f. sp. tritici, is a serious wheat fungal disease, causing significant annual yield losses worldwide. The Chinese wheat cultivar Chuanyu 16 has shown good adult-plant resistance (APR) to stripe rust in Sichuan province, a hotspot for stripe rust epidemics. Chuanyu 16 was crossed with Chuanyu 12 and Chuanmai 32. Two populations, each with 140 recombinant inbred lines (RILs), were developed by single-seed descent, and used for quantitative trait locus (QTL) mapping. Field trials were conducted in Chengdu and Yaan from 2005 to 2008, providing stripe rust reaction data for six environments. Seven hundred and thirty one simple sequence repeat (SSR) markers were screened for association with stripe rust reaction, initially through bulked segregant analysis (BSA). Three QTLs for stripe rust resistance derived from Chuanyu 16 were detected in the first cross. They were detected by inclusive composite interval mapping (ICIM) and designated QYr.caas-1BL.1, QYr.caas-1BL.2 and QYr.caas-2AS. They explained 6.0 - 12.8\%, 4.5 $5.8 \%$ and $14.9-43.0 \%$, respectively, of the phenotypic variance across environments. One digenic epistatic QTL between QYr.caas-1BL.2 and QYr.caas-2AS explained 4.3 - 10.4\% of the phenotypic variance. QYr.caas-2AS was also detected in Chuanmai 32/Chuanyu 16, explaining $27.9-57.2 \%$ of the phenotypic variance across six environments. This QTL showed a major effect against stripe rust in Chuanyu 16, and was located in a similar position to Yr17. Specific markers indicated the presence of a segment from chromosome $2 \mathrm{~N}$ of Triticum ventricosum that carries $\operatorname{Yr} 17$. Despite the lack of evidence for $\operatorname{Yr} 17$ in Chuanyu 16 based on pedigree, and inconsistencies in stripe rust response relative to a near-isogenic reference stock with the gene, we concluded that QYr.caas-2AS is Yr17. QYr.caas-1BL.1 and QYr.caas-1BL.2 showed minor effects for APR against stripe rust. QYr.caas-1BL.1 is probably a new gene for APR to stripe rust.
\end{abstract}

Keywords: APR, Microsatellites, Quantitative trait locus, Triticum aestivum, Yellow rust

\title{
1. Introduction
}

Stripe rust, caused by Puccinia striiformis f. sp. tritici (Pst), is a devastating wheat disease worldwide. Breeding and utilizing resistant wheat cultivars are the most economic and environmentally friendly way to control the disease (Line 2002; Chen 2005). Wheat rust resistance was conferred by race-specific major resistance or adult-plant resistance genes. The race-specific major gene resistance is usually effective at both the seedling and adult-plant stages, eliciting a hypersensitive response upon infection by a pathogen race possessing a corresponding virulence allele (McIntosh et al., 1995). When deployed in agriculture this type of resistance is overcome by virulent races. In contrast, adult-plant resistance, horizontal, slow-rusting or partial resistance is usually race non-specific, and expressed by increased latent period and reduced uredinial size, infection frequency and spore production (Caldwell 1968; Ohm and Shaner 1976; Parlevliet 1975). Wheat genotypes with this kind of resistance are usually susceptible at the seedling stage, and resistance develops as the plants approach the flowering stage and as temperatures increase. This kind of resistance is called adult-plant resistance (APR).

Several genetic analyses of APR show that resistance is conferred by the additive effects of a few genes (Singh and Rajaram 1994; Navabi et al., 2004; Singh et al., 2005). Epistatic effects of such genes have also been reported (Lin and Chen 2009; Lu et al., 2009). Field assessments at the adult plant stage in multiple environments are needed for genetic analysis of APR. To date, about 48 genes for stripe rust resistance are catalogued and 36 are temporarily designated. Among them, $Y r 11-Y r 14, \operatorname{Yr} 16, \operatorname{Yr} 18, \operatorname{Yr} 29, \operatorname{Yr} 30, \operatorname{Yr} 36, \operatorname{Yr} 39$, $Y r 46$ and $Y r 48$ are described as APR genes, but not all are race non-specific, whereas the others are race-specific major resistance genes (Chen 2005; McIntosh et al., 2010). About 77 QTLs for APR to stripe rust have been mapped on 17 of the 21 wheat chromosomes except for 1A, 1D, 3A and 7A (He et al. ,2011).

Sichuan province is a hotspot for stripe rust epidemics in China, and various new virulent races arose or emerged in this area. From previous reports (Wan et al., 2004, 2007), only Yr5, Yr10, Yr15, Yr24 and Yr26 confer resistance to the prevalent Chinese Pst race CYR 32. Cultivar Chuanyu 16 was released in 2002 with high resistance to stripe rust at the adult stage in the field. In the same year a widespread stripe rust epidemic affected about 6.6 million hectares of wheat, especially in Sichuan province. Most wheat cultivars with the widely used Yr9 or Fan 6 resistance genes were affected by Pst races CYR 31 and CYR 32 (Wan et al., 2004). With the change in predominant races from CYR 30 to CYR 32 and CYR 33 over the past nine years, there was an increase in disease rating on cv. Chuanyu 16, but the increase was much less than on other genotypes with the so 
called Fan 6 resistance. Cv. Chuanyu 16 has APR genes conferring moderate adult plant protection with stripe rust severity lower than $30 \%$ under average conditions and lower than $50 \%$ in severe epidemic conditions. With this level of resistance in combination with excellent agronomic traits, cv. Chuanyu 16 became a leading breeding parent in Sichuan. However, the chromosomal location and genetic effects of its APR genes remained unknown. The objective of this study was to identify the QTLs for APR to stripe rust in cv. Chuanyu 16 in a RIL population derived from a cross between cv. Chuanyu 16 and susceptible cultivar Chuanyu 12, and to validate the QTLs with a second RIL population derived from a cross between cv. Chuanmai 32 and cv. Chuanyu 16.

\section{Materials and methods}

\subsection{Plant materials}

Cv. Chuanyu 16, developed by the Chengdu Institute of Biology, Chinese Academy of Science, and released in Sichuan in 2002, is highly susceptible to Pst race CYR 32 at the seedling stage, and resistant to moderately resistant at the adult-plant stage, with stripe rust severity lower than $30 \%$ in normal epidemic years and lower than $50 \%$ in heavy epidemic years. Cv. Chuanyu 12, a $1 \mathrm{~B} \cdot 1 \mathrm{R}$ line with $\mathrm{Yr} 9$, developed by the same institute and released in Sichuan in 1992, is highly susceptible to Pst races CYR 31 and CYR 32 at both the seedling and adult-plant stages, although it was originally resistant when released. Cv. Chuanmai 32, developed by the Crop Research Institute, Sichuan Academy of Agricultural Science and released in 2001, is moderately resistant to Pst races CYR 31 and CYR 32 at the adult plant stage, with stripe rust severity nearly $30 \%$. One population of 140 $\mathrm{F}_{7}$ RILs, derived from cv. Chuanyu 16/cv. Chuanyu 12, was used for QTL mapping, and a second population, also with 140 RILs, derived from cross cv. Chuanmai 32/cv. Chuanyu 16 was used for validating the QTLs and linked molecular markers.

\subsection{Field trials and phenotypic characterization of stripe rust response}

The two RIL populations were evaluated for stripe rust severity at sites near Chengdu in 2005, 2006, and 2007 and Yaan in 2006, 2007 and 2008 cropping seasons, providing data for six environments. Chengdu $\left(103.85^{\circ} \mathrm{E}\right.$, $\left.30.81^{\circ} \mathrm{N}\right)$ and Yaan $\left(103.11^{\circ} \mathrm{E}, 29.95^{\circ} \mathrm{N}\right)$ are located about $150 \mathrm{Km}$ apart in Sichuan. Chengdu is located in the central region of Sichuan basin where heavy stripe rust epidemics occurred every two or three years, while Yaan is in the southwestern region of Sichuan basin where heavy stripe rust epidemics occurred every year. The experimental plots were completely randomized with three replications in Chengdu 2005 and 2006, and two replications in Yaan 2006 and Chengdu 2007, and one replication in Yaan 2007 and 2008. Plots consisted of single rows $1.5 \mathrm{~m}$ in length and $30 \mathrm{~cm}$ between rows, with approximately 50 seeds sown in each row. The susceptible parent cv. Chuanyu 12 was included every tenth row as a susceptible check and as inoculum sources, and was also planted around the test area to ensure ample inoculum in spring.

Yaan is a hotspot for stripe rust epidemics. The field trials in the present work depended on natural epidemics at Yaan. In Chengdu, Pst race CYR 32 on seedlings of cv. Chuanyu 12 was introduced into the trial at the three-leaf stage in each season. Stripe rust severity after anthesis was recorded on line basis as visual estimates of percentage leaf area infected. Around April 15-20 in Chengdu and April 20-25 in Yaan, the stripe rust severity in the susceptible parent cv. Chuanyu 12 displayed between $85 \%$ and $100 \%$. The data from readings at that time were the maximum disease severity (MDS).

\subsection{Statistical analysis}

The SAS statistical package (SAS Institute, Cary, NC, USA) was used to conduct an analysis of variance (ANOVA) for estimating genetic and environmental effects of lines, environments and line $\times$ environment interactions using data from Chengdu 2005, 2006 and 2007, and Yaan 2006 in which two or three replications were carried out in the field trials. The ANOVA results were used to estimate the heritability $\left(h^{2}\right)$ calculated by the formula $h^{2}=\mathrm{V}_{\mathrm{l}} /\left(\mathrm{V}_{\mathrm{l}}+\mathrm{V}_{\mathrm{le}}+\mathrm{V}_{\mathrm{e}}\right)$, where $\mathrm{V}_{\mathrm{l}}, \mathrm{V}_{\mathrm{le}}$ and $\mathrm{V}_{\mathrm{e}}$ are the variance component estimates of lines, line $\times$ environment interaction and environments (Basford et al. 2004, Yang. 2007), respectively. As replications were not the same in the field trial every year, the variance components $\left(V_{l}, V_{l e}\right.$ and $\left.V_{e}\right)$ were estimated by REML model in SAS software. Phenotypic correlation coefficients among different environments for MDS were calculated on a mean basis using the Microsoft Excel analytical tool.

\subsection{Molecular marker analysis}

The CTAB method (Sharp et al., 1988) was used to extract genomic DNA from young leaves of the parents and $\mathrm{F}_{7}$ RILs. SSR analysis, including PCR, polyacrylamide gel electrophoresis and gel staining, followed Li et al. (2006) and Bassam et al. (1991). Cv. Chuanyu 16, cv. Chuanyu 12 and two bulks were screened with 731 SSR markers, including 561 pairs of primers from the Beltsville Agriculture Research Center (BARC) (Song et al., 
2002), 114 pairs of primers from Gatersleben Wheat Microsatellite (GWM) (Röder et al., 1998), 38 pairs of primers from Wheat Microsatellite Consortium (WMC) (Gupta et al., 2003), 12 pairs of primers from the Clermont Ferrand D-genome (CFD) set (Guyomarc'h et al., 2002), 5 pairs of primers from the Clermont Ferrand A-genome (CFA) set (Sourdille et al., 2004) and Xcewm32 (Zhu et al., 2010). Two markers linked with Yr17, VENTRIUP-LN2 and CAPS marker URIC-LN2-Dpn II (Helguara et al., 2003), were also used.

\subsection{Bulked segregant analysis}

Based on averaged disease severities from six environments, five lines with the highest levels of resistance were selected to make a resistant bulk and five with the highest levels of susceptibility were used to make a susceptible bulk. After adjusting DNA concentrations, equal amounts of DNA from each line were mixed to make the respective bulks. SSRs were selected to genotype the 10 most resistant and 10 most susceptible lines, respectively, when the same patterns of SSR polymorphism were observed between the resistant and susceptible parents, and between the resistant and susceptible bulks. Those SSRs showing linkage with stripe rust reaction were used to genotype the entire population. Additional markers for enriching the chromosomal regions linked to resistance genes were selected from published wheat consensus maps (http://www.shigen.nig.ac.jp/wheat; http://wheat.pw.usda.gov; Somers et al. 2004) and tested for polymorphism between the parents and between bulks. Those showing polymorphism were also used to genotype the entire population for linkage analysis.

\subsection{Map construction and QTL detection}

QTL mapping was based on the averaged MDS in each environment, and also the averaged data across all environments. Software IciMapping 3.0 was employed for construction of a genetic linkage map (Wang et al 2010). Map distances between markers were calculated using the Kosambi (1944) mapping function. The positions of detected QTLs and digenic interactions between non-allelic QTLs were determined by inclusive composite interval mapping (ICIM) using IciMapping 3.0 (Li et al. 2007, 2008). A logarithm of odds (LOD) of 2.0 was set to declare significance of QTLs. Each QTL was represented by a 20-centimorgan (cM) interval with the local LOD maximum as central point. QTL with overlapping $20 \mathrm{cM}$ intervals from different environments were considered to be the same. QTL effects were estimated as the proportion of phenotypic variance explained (PVE) by the QTL. The chromosomal assignments of the linkage group were based on published wheat maps (Somers et al. 2004), Graingenes (http://wheat.pw.usda.gov) and the Komugi integrated wheat consensus maps (http://www.shigen.nig.ac.jp/wheat).

\subsection{Validation of QTLs and their linked molecular markers}

The 33 markers linked to QTLs identified in the cv. Chuanyu 16/cv. Chuanyu 12 RIL population were used to screen the parents, the 10 most resistant and 10 most susceptible lines of the cv. Chuanmai 32/cv. Chuanyu 16 population, and 25 of them showing associations with stripe rust resistance were used to genotype the entire population, and to construct a framework map for QTL analysis in this population.

\section{Results}

\subsection{Phenotypic analysis of MDS, and their correlations and heritabilities}

In the cv. Chuanyu $16 / \mathrm{cv}$. Chuanyu 12 RIL population, the MDS scores were significantly correlated across the six environments, with correlation coefficients ranging from 0.49 to $0.78(P<0.0001)$. The frequency distributions of MDS showed continuous distributions in the different environments (Fig. 1). The mean MDS of cv. Chuanyu 16 and cv. Chuanyu 12 were $48 \%$ and $99 \%, 10 \%$ and $77 \%, 20 \%$ and $91 \%$ in Chengdu 2005, 2006 and 2007, respectively, and $20 \%$ and $98 \%, 16 \%$ and $100 \%$, and $14 \%$ and $89 \%$ in Yaan 2006, 2007 and 2008, respectively. The averaged MDS of the 140 RILs across all six environments was $54.0 \%$, ranging from 0.0 to $100.0 \%$. The heritability of MDS was 0.68 based on disease severity averaged from four environments, viz. Chengdu 2005, 2006, 2007 and Yaan 2006. The ANOVA confirmed significant variance $(P<0.0001)$ among RILs in the population (Table 1).

In cv.Chuanmai $32 / \mathrm{cv}$. Chuanyu 16, MDS scores were significantly correlated across the six environments, with correlation coefficients ranging from 0.47 to $0.80(P<0.0001)$. The frequency distributions of stripe rust MDS showed continuous distributions over the different environments (Fig. 2). The mean MDS of cv. Chuanmai 32 and cv. Chuanyu 16 were $26 \%$ and $48 \%, 20 \%$ and $10 \%, 38 \%$ and 20\% in Chengdu 2005, 2006 and 2007, respectively, and $14 \%$ and $20 \%, 10 \%$ and $16.0 \%, 15 \%$ and $14 \%$ in Yaan 2006, 2007 and 2008, respectively. The averaged MDS of the 140 RILs across six environments was $30.0 \%$, ranging from 0.9 to $83.3 \%$. The heritability of MDS was 0.76 based on the disease severity averaged from four environments, viz. Chengdu 2005, 2006, 2007 and Yaan 2006. ANOVA confirmed significant variance $(P<0.0001)$ among RILs in the population 
(Table 1).

\subsection{The overall SSR polymorphisms between Chuanyu 16 and Chuanyu 12}

Among 731 SSR markers tested in Chuanyu 16 /Chuanyu 12 population, 355 SSRs showed polymorphisms between two parents; 217 SSRs displayed polymorphisms between the resistant and susceptible bulks; of them, 73 SSRs were used to genotype the 10 most resistant and 10 most susceptible lines; and finally 58 SSRs were used to genotype 140 RILs of cv. Chuanyu $16 / \mathrm{cv}$. Chuanyu 12 population. The genotyping results of the SSR markers, VENTRIUP-LN2 and a CAPS marker URIC-LN2-Dpn II were used to construct linkage map for QTL detection.

\subsection{QTLs for APR to stripe rust in the Chuanyu 16/Chuanyu 12 RIL population}

Based on the MDS data for the cv. Chuanyu 16/cv. Chuanyu 12 population, three QTLs for stripe rust resistance were detected by ICIM across six environments (Table 2; Fig. 3). According to wheat consensus maps (http://www.shigen.nig.ac.jp/wheat; http://wheat.pw.usda.gov; 42), two QTLs were located on the long arm of chromosome $1 \mathrm{~B}$, and one was located on the short arm of chromosome $2 \mathrm{~A}$. They were designated QYr.caas-1BL.1, QYr.caas-1BL.2 and QYr.caas-2AS, and all came from the resistant parent Chuanyu 16.

The most consistent locus with the largest effect found in all environments was QYr.caas-2AS, located on the short arm of chromosome 2A. It was flanked by Xcfd36 and Xwmc598, and the peak LOD score was close to Xgwm 497 and URIC-LN2-Dpn II. This QTL explained 18.1\%, 37.3\%, 27.8\%, 43.0\%, $14.9 \%$ and $24.7 \%$ of the phenotypic variance in Chengdu 2005, 2006, 2007 and Yaan 2006, 2007, 2008, with additive effects of 10.8, 15.4, 15.4, 22.4, 14.3 and 15.8, respectively (Table 2 and Fig. 3B). The phenotypic variance was as high as $32.2 \%$ for QTL computed by averaged MDS across all environments. The second QTL, QYr.caas-1BL.1, located on chromosome 1BL, and flanked by the SSR loci Xbarc61 and Xwmc134, explained $6.0 \%$ and $12.8 \%$ of the phenotypic variance in Chengdu 2006 and Yaan 2007, with additive effects of 6.2 and 13.3, respectively, and the phenotypic variance explained for the averaged MDS across all environments was $11.2 \%$ (Table 2 and Fig. 3A). The third QTL, QYr.caas-1BL.2, also on chromosome 1BL, was detected in the marker interval Xgwm818 Xgwm259. This QTL explained 5.5\%, 4.5\% and 5.1\% of the phenotypic variance in Chengdu 2005, Chengdu 2007 and Yaan 2007, with additive effects of 6.0, 6.3 and 8.5, respectively, and the phenotypic variance explained by the QTL for the averaged MDS across all environments was 5.8\% (Table 2 and Fig. 3A).

\subsection{Validation of QTLs in the Chuanmai 32/Chuanyu 16 RIL population}

Based on the MDS and molecular marker data, QYr.caas-2AS was also detected in the cv. Chuanmai 32/cv. Chuanyu 16 RIL population with significant effects across all environments (Table 2). The QTL was flanked by Xwmc407 and VENTRIUP-LN2, and explained 35.5\%, 57.2\%, 49.8\%, 45.9\%, 29.0\%, 27.9\% and 56.9\% of the phenotypic variance in Chengdu 2005, 2006, 2007, Yaan 2006, 2007, 2008 and in the averaged MDS, with additive effects of 14.1, 16.2, 18.8, 19.3,11.2, 11.1 and 15.1, respectively. QTLs QYr.caas-1BL.1 and QYr.caas-1BL.2 were not found in this population, possibly because there was no allelic variation between the parents at these loci (Fig. 3C).

\subsection{Epistatic effects between QTLs}

Among the three QTLs in cv. Chuanyu 16, an epistatic interaction between QYr.caas-1BL.2 and QYr.caas-2AS was detected in all environments and averaged MDS of all environments, explaining from 4.3 to $10.4 \%$ of the phenotypic variance (Table 3).

\section{Discussion}

$\mathrm{Cv}$. Chuanyu 16 is susceptible to Pst race CYR 32 at the seedling stage and resistant or moderately resistant at the adult stage, with a typical of APR to stripe rust (Caldwell 1968; Ohm and Shaner 1976; Parlevliet 1975). QYr.caas-1BL.1, QYr.caas-1BL.2 and QYr.caas-2AS were detected in the cv. Chuanyu 16/cv. Chuanyu 12 RIL population, and $Q Y$ r.caas- $2 A S$ was validated in the cv. Chuanmai 32/cv. Chuanyu 16 population. The present QTL analysis determined the locations and effects of APR genes for stripe rust resistance in cv. Chuanyu 16.

Christiansen et al. (2006) located QTL.2AS for stripe rust resistance on the short arm of chromosome 2A. This gene, located $2 \mathrm{cM}$ from the SSR locus Xwmc 407 in progenies of Kris/Wasmo and Kris/Deben, was assumed to be Yr17. Chhuneja et al. (2008) detected QYrtm.pau-2A in T. monococcum acc. pau14087 in a 3.6-cM interval of Xwmc407 and Xwmc170 on chromosome 2A. Dedryvere et al. (2009) assumed QYrinra-2AS.2 to be the same as Yr17 in a Renan/Revital population. $\operatorname{Yr} 17$ is in a $\operatorname{Lr} 37-Y r 17-S r 38$ cluster, located within a segment of the short arm of chromosome $2 \mathrm{~N}$ of Triticum ventricosum. This segment was translocated to the short arm of bread wheat chromosome 2A, and introgressed into the winter bread wheat 'VPM1' (Maia 1967; Bariana and McIntosh 1993; 
McIntosh et al., 1995). Two markers, URIC-LN2-Dpn II and VENTRIUP-LN2, were developed to characterize the 2NS/2AS chromosome segment (Helguera et al., 2003). In the present study, the 2NS-specific PCR products of VENTRIUP-LN2 (259 bp) and URIC-LN2 (285 bp) were amplified in cv. Chuanyu 16, and both markers were closely linked in coupling with QYr.caas- $2 A S$ in the cv. Chuanyu $16 / \mathrm{cv}$. Chuanyu 12 and cv. Chuanmai $32 / \mathrm{cv}$. Chuanyu 16 populations. This indicated that QYr.caas-2AS was located in a $2 \mathrm{NS}$ segment, and that it was likely to be $\operatorname{Yr} 17$. However, this deduction is difficult to reconcile in relation to the response of a $\mathrm{Yr} 17 / 6^{*}$ Avocet $\mathrm{S}$ near-isogenic line (NIL) used as a reference for $\mathrm{Yr} 17$. According to field tests in Chengdu and Yaan from 2001 to 2010, the $\operatorname{Yr} 17 / 6^{*}$ Avocet S NIL was resistant in all years except 2002 and 2007. cv. Chuanyu 16 and Yr17/6*Avocet S NIL were scored susceptible to CYR 32 at seedling tests, but the responses of lines with $\mathrm{Yr} 17$ are known to be environmentally sensitive even in greenhouse tests (Wellings personal communication). At the adult-plant stage in the field, the infection type of $Y r 17 / 6^{*}$ Avocet S NIL was 0 ; and the stripe rust severities were lower than 5\%, whereas the corresponding scores for cv. Chuanyu 16 were infection type $4(0-4$ scale) and disease severities was nearly $30 \%$ in 2009 and 2010. It is now known that $Y r 17 / 6^{*}$ Avocet S NIL along with some other Avocet S NILs carry $\operatorname{Yr} 18$ in addition to the designated genes. Thus it is possible that the lower response of Yr17/6*Avocet S NIL compared with cv. Chuanyu 16 was due to the presence of $\operatorname{Yr} 18$ in combination with $\operatorname{Yr} 17$. cv. Chuanyu 16 was derived from the cross 30020/Miannong 4//Jinmai 30; cv. Miannong 4 and cv. Jinmai 30 are cultivars susceptible to Pst races CYR 31, CYR 32 and CYR 33. The other parent, 30020, a breeding line at the Chengdu Institute of Biology, was probably the source of the resistance to stripe rust in cv. Chuanyu 16. This line has a complicated background, being derived from Kavkaz, Fan 6, Fan 7, NPFP, Aurora and a hexaploid triticale, but VPM1 or a derivative is not known to be involved. An alternative hypothesis that a $2 \mathrm{~N}$ segment in $\mathrm{cv}$. Chuanyu 16 carries a gene different from $\operatorname{Yr} 17$ or carries a wheat gene completely linked in coupling with a $2 \mathrm{~N}$ segment appears highly unlikely.

Although both QYr.caas-1BL.1 and QYr.caas-1BL.2 were mapped on 1BL, they were located to different marker intervals of Xbarc61 - Xwmc134 and Xgwm818 - Xgwm259, respectively. According to the wheat composite map 2004-1B (http://wheat.pw.usda.gov), the distance between Xbarc61 and Xgwm259 is $31 \mathrm{cM}$, and that between Xwmc134 and Xgwm259 is $47 \mathrm{cM}$. In addition, the two QTLs were not overlapping, thus QYr.caas-1BL.1 and QYr.caas-1BL.2 were considered to be different. To date, 11 race-specific stripe rust resistance genes, viz. $\operatorname{Yr} 3$ (first described in Lupton and Macer 1962), $\operatorname{Yr} 9$ (Macer 1975), $Y r 10$ (Macer 1975), $\operatorname{Yr} 15$ (Gerechter-Amitai et al., 1989), Yr21 (Chen et al., 1995), Yr24 (McIntosh et al., 1998), Yr26 (Ma et al., 2001), YrCH42 (Li et al., 2006), YrH52 (Peng et al., 2000), Yrchk (Fanghui Liu et al., 2007) and Yrexpl (Lin and Chen 2008) were located on chromosome 1B. The two QTLs found in the present study are not likely to be the same as any of these genes. Four of five previously reported QTLs for stripe rust APR on 1B, QYr.cimmyt-1BL (William et al., 2006), QYr.csiro-1BL (Rosewarne et al., 2008), QPst.jic-1B (Melichar et al., 2008) and QYr.saas-1BL (Zhu et al., 2010), could be $\operatorname{Yr} 29$ (summarized in Table 4). This gene, closely linked to SSR locus Xgwm259, has a relatively large effect on stripe rust response (William et al., 2003; Singh et al., 1998) and co-segregates with csLV46 (personal communication with Dr. Evans Lagudah). QYrex.wgp-1BL, flanked by Xwmc631 and Xgwm268 in Express, was located at a different position from $\operatorname{Yr} 29$ on $1 \mathrm{BL}$, and accounted for $12.4-15.9 \%$ of the phenotypic variance (Lin and Chen 2009). QYr.caas-1BL.1 in the present study was flanked by markers Xbarc61 and Xwmc134. The distance between Xwmc134 and Xgwm259 is about $47 \mathrm{cM}$, and that between Xwmc134 and Xwmc631 is about 10 cM (http://wheat.pw.usda.gov; Somers et al. 2004). This indicates that QYr.caas-1BL.1 is not only different from Yr29, but is also different from QYrex.wgp-1BL. It could be a new QTL for APR to stripe rust. QYr.caas-1BL.2 found in the present study was flanked by Xgwm818 and Xgwm259, in a similar location to $\operatorname{Yr} 29$ and the four QTLs previously reported. However, the Yr29-specific band from csLV46 was not amplified in cv. Chuanyu 16. In addition, QYr.caas-1BL.2 confers a much lower level of resistance compared to that reported for Yr 29 .

\section{Conclusion}

APR to stripe rust in cv. Chuanyu 16 were conferred by three QTLs designated QYr.caas-1BL.1, QYr.caas-1BL.2 and QYr.caas-2AS. QYr.caas-2AS with a major effect on APR was located in a similar position to Yr17, and since it was associated with markers specific for a $2 \mathrm{NS}$ chromosome introgression, we suggest it is $\operatorname{Yr} 17$ despite inconsistencies in pedigree and stripe rust response data. QYr.caas-1BL.1 and QYr.caas-1BL.2 showed minor effects on stripe rust response. QYr.caas-1BL.1 was likely to be a new gene; QYr.caas-1BL.2 could be different from $\mathrm{Yr}_{2} 9$ although it mapped to a similar position.

\section{Acknowledgements}

The authors are grateful to Prof. R. A. McIntosh, Plant Breeding Institute, University of Sydney, for the critical review of this manuscript. This study was supported by the National Science Foundation of China 
(30821140351), International Collaboration Project from the Ministry of Agriculture (2011-G3) and an earmarked fund for Modern Agro-industry Technology Research System.

\section{References}

Bariana, H.S., \& McIntosh, R.A. (1993). Cytogenetic studies in wheat. XV. Location of rust resistance genes in VPM1 and their genetic linkage with other disease resistance genes in chromosome 2A. Genome, 36, 476-482. http://dx.doi.org/10.1139/g93-065

Basford, K.E., Federer, W.T., \& DeLacy, I.H. (2004). Mixed model formulations for multi-environment trials. Agronomy. Journal, 96, 143-147. http://dx.doi.org/10.2134/agronj2004.1430

Bassam, B.J., Caetano-Anollés, G., \& Gresshoff, P.M. (1991). Fast and sensitive silver staining of DNA in polyacrylamide gels. Anaytical. Biochemistry, 196, 80-83. http://dx.doi.org/10.1016/0003-2697(91)90120-I

Caldwell, R.M. (1968). Breeding for general and/or specific plant disease resistance. In K.W. Finlay, \& K.W. Shepherd (Eds.). Proc. Third Int. Wheat Genet. Symp (pp. 263-272). Canberra: Australian Academy of Science.

Chen, X.M. (2005). Epidemiology and control of stripe rust (Puccinia striiformis f. sp. tritici) on wheat. Canadina Journal of Plant Pathology, 27, 314-337. http://dx.doi.org/10.1080/07060660509507230

Chen, X.M., Jones, S.S., \& Line, R.F. (1995). Chromosomal location of genes for stripe rust resistance in spring wheat cultivars Compair, Fielder, Lee and Lemhi and interactions of aneuploid wheats with races of Puccinia striiformis. Phytopathology, 85, 375-381. http://dx.doi.org/10.1094/phyto-85-375

Chhuneja, P., Kaur, S., Garg, T., Ghai, M., Kaur, S., Prashar, M., Bains, N.S., Goel, R.K., Keller, B., Dhaliwal, H.S., \& Singh, K. (2008). Mapping of adult plant stripe rust resistance genes in diploid A genome wheat species and their transfer to bread wheat. Theoretical and Applied Genetics, 116, 313-324. http://dx.doi.org/10.1007/s00122-007-0668-0

Christiansen, M.J., Feenstra, B., Skovgaard, I.M., \& Andersen, S.B. (2006). Genetic analysis of resistance to yellow rust in hexaploid wheat using a mixture model for multiple crosses. Theoretical and Applied Genetics, 112, 581-591. http://dx.doi.org/10.1007/s00122-005-0128-7

Dedryver, F., Paillard, S., Mallard, S., Robert, O., Trottet, M., Negre, S., Verplancke, G., \& Jahier, J. (2009). Characterization of genetic components involved in durable resistance to stripe rust in the bread wheat 'Renan'. Phytopathology, 99, 968-973. http://dx.doi.org/10.1094/PHYTO-99-8-0968

Gerechter-Amitai, Z.K., Silfhout, C.H., Grama, A., \& Kleitman, F. (1989). Yr15-a new gene for resistance to Puccinia striiformis in Triticum dicoccoides sel. G-25. Euphytica, 43, 187-190. http://dx.doi.org/10.1007/BF00037912

Gupta, P.K., Rustgi, S., Sharma, S., Singh, R., Kumar, N., \& Balyan, H.S. (2003). Transferable EST-SSR markers for the study of polymorphism and genetic diversity in bread wheat. Molecular Genetics Genomics, 270, 315-323. http://dx.doi.org/10.1007/s00438-003-0921-4

Guyomarc'h, H., Sourdille, P., Charmet, G., Edwards, K., \& Bernard, M. (2002). Characterisation of polymorphic microsatellite markers from Aegilops tauschii and transferability to the D-genome of bread wheat. Theoretical and Applied Genetics, 104, 1164-1172. http://dx.doi.org/10.1007/s00122-001-0827-7

Helguera, M., Khan, I.A., Kolmer, J., Lijavetzky, D., Liu, Z.Q., \& Dubcovsky, J. (2003). PCR assays for the Lr37-Yr17-Sr38 cluster of rust resistance genes and their use to develop isogenic hard red spring wheat lines. Crop Science, 43, 1839-1847. http://dx.doi.org/10.2135/cropsci2003.1839

He, Z.H., Lan, C.X., Chen, X.M., Zou, Y.C., Zhuang, Q.S., \& Xia, X.C. (2011). Progress and perspective in research of adult-plant resistance to stripe rust and powdery mildew in wheat. Scientia Agricultura Sinica., 44, 2193-2215 (in Chinese with English abstract). http://dx.doi.org/10.3864/j.issn.0578-1752.2011.11.001

Kosambi, D.D. (1944). The estimation of map distance from recombination values. Annals of Eugenics, 12, $172-175$.

Li, G.Q., Li, Z.F., Yang, W.Y., Zhang, Y., He, Z.H., Xu, S.C., Singh, R.P., Qu, Y.Y., \& Xia, X.C. (2006). Molecular mapping of stripe rust resistance gene $\mathrm{YrCH} 42$ in Chinese wheat cultivar Chuanmai 42 and its allelism with Yr24 and Yr26. Theoretical and Applied Genetics, 112, 1434-1440. http://dx.doi.org/10.1007/s00122-006-0245-y

Li, H.H., Ye, G.Y., \& Wang, J.K. (2007). A modified algorithm for the improvement of composite interval mapping. Genetics, 175, 361-374. http://dx.doi.org/10.1534/genetics.106.066811 
Li, H.H., Ribaut, J.-M., Li, Z.L., \& Wang, J.K. (2008). Inclusive composite interval mapping (ICIM) for digenic epistasis of quantitative traits in biparental populations. Theoretical and Applied Genetics, 116, 243-260. http://dx.doi.org/10.1007/s00122-007-0663-5

Lin, F., \& Chen, X.M. (2008). Molecular mapping of genes for race-specific overall resistance to stripe rust in wheat cultivar Express. Theoretical and Applied Genetics, 116, 797-806. http://dx.doi.org/10.1007/s00122-008-0713-7

Lin, F., \& Chen, X.M. (2009). Quantitative trait loci for non-race-specific, high-temperature adult-plant resistance to stripe rust in wheat cultivar Express. Theoretical and Applied Genetics, 118, 631-642. http://dx.doi.org/10.1007/s00122-008-0894-0

Line, R.F. (2002). Stripe rust of wheat and barley in North America: a retrospective historical review. Annual Review of Phytopathology, 40, 75-118. http://dx.doi.org/10.1146/annurev.phyto.40.020102.111645

Liu, F.H., Niu, Y.C., Deng, H., \& Tan, G.J. (2008). Mapping of major stripe rust resistance gene in Chinese native wheat variety Chike using miscrosatellite markers. Journal of Genetics \& Genomics, 34, 1123-1130. http://dx.doi.org/10.1016/s1673-8527(07)60128-3

Lu, Y.M., Lan, C.X., Liang, S.S., Zhou, X.C., Liu, D., Zhou, G., Lu, L.Q., Jing, J.X., Wang, M.N., Xia, X.C., \& He, Z.H. (2009). QTL mapping for adult-plant resistance to stripe rust in Italian common wheat cultivars Libellula and Strampelli. Theoretical and Applied Genetics, 119, 1349-1359. http://dx.doi.org/10.1007/s00122-009-1139-6

Lupton, F.C.H., \& Macer, R.C.F. (1962). Inheritance of resistance to yellow rust (Puccinia glumarum Erikss. and Henn.) in seven varieties of wheat. Transactions of the British Mycological Society, 45, 21-45. http://dx.doi.org/10.1016/s0007-1536(62)80032-1

Ma, J.X., Zhou, R.H., Dong, Y.S., Wang, L.F., Wang, X.M., \& Jia, J.Z. (2001). Molecular mapping and detection of the yellow rust resistance gene $\mathrm{Yr} 26$ in wheat transferred from Triticum turgidum L. using microsatellite markers. Euphytica, 120, 219-226. http://dx.doi.org/10.1023/A:1017510331721

Macer, R.C.F. (1975). Presidential address: Plant pathology in a changing world. Transactions Of the British Mycological Society, 65, 351-374. http://dx.doi.org/10.1016/s0007-1536(75)80032-5

Maia, N. (1967). Obtention des bles tendres resistants au pietin-verse par croisements interspecifiques bles $\times$ Aegilops. Comptes Rendus de l'Academie d'Agriculture de France, 53, 149-154.

McIntosh, R.A., Hart, G.E., Devos, K.M., Rogers, J., \& Gale, M.D. (1998). Catalogue of gene symbols for wheat: 1998 supplement. Annual Wheat Newsletter, 44.

McIntosh, R.A., Dubcovsky, J., Rogers, W.J., Morris, C., Appels, R., \& Xia, X.C. (2010). Catalogue of gene symbols for wheat: 2010 supplement. [Online] Available: http://www.shigen.nig.ac.jp/wheat/komugi/genes/macgene/supplement2010.pdf.

McIntosh, R.A., Wellings, C.R., \& Park, R.F. (1995). Wheat Rusts, an Atlas of Resistance Genes. CSIRO, Melbourne, Australia.

Melichar, J.P.E., Berry, S., Newell, C., MacCormack, R., \& Boyd, L.A. (2008). QTL identification and microphenotype characterization of the developmentally regulated yellow rust resistance in the UK wheat cultivar Guardian. Theoretical and Applied Genetics, 117, 391-399. http://dx.doi.org/doi:10.1007/s00122-008-0783-6

Navabi, A., Singh, R.P., Tewari, J.P., \& Briggs, K.G. (2004). Inheritance of high levels of adult-plant resistance to stripe rust in five spring wheat genotypes. Crop Science., 44, 1156-1162. http://dx.doi.org/10.2135/cropsci2004.1156

Ohm, H.W., \& Shaner, G.E. (1976). Three components of slow-leaf rusting at different growth stages in wheat. Phytopathology, 66, 1356-1360. http://dx.doi.org/10.1094/phyto-66-1356

Parlevliet, J.E. (1979). Components of resistance that reduce the rate of epidemic development. Annual Review of Phytopathology, 17, 203-222. http://dx.doi.org/10.1146/annurev.py.17.090179.001223

Peng, J.H., Fahima, T., Röder, M.S., Huang, Q.Y., Dahan, A., Li, Y.C., Grama, A., \& Nevo, E. (2000). High-density molecular map of chromosome region harboring stripe-rust resistance genes $\mathrm{YrH}_{52}$ and $\mathrm{Yr} 15$ derived form wild emmer wheat, Triticum dicoccoides. Genetica, 109, 199-210. http://dx.doi.org/10.1023/A:1017573726512 
Robert, O., Abelard, C., \& Dedryver, F. (1999). Identification of molecular markers for the detection of the yellow rust resistance gene $Y r 17$ in wheat. Molecular. Breeding, 5, 167-175. http://dx.doi.org/10.1023/A:1009672021411

Rosewarne, G.M., Singh, R.P., Huerta-Espino, J., \& Rebetzke, G.J. (2008). Quantitative trait loci for slow-rusting resistance in wheat to leaf rust and stripe rust identified with multi-environment analysis. Theoretical and Applied Genetics, 116, 1027-1034. http://dx.doi.org/10.1007/s00122-008-0736-0

Röder, M.S., Korzun, V., Wendehake, K., Plaschke, J., Tixier, M.-H., Leroy, P., \& Ganal, M.W. (1998). A microsatellite map of wheat. Genetics, 149, 2007-2023.

Sharp, P.J., Kreis, M., Shewry, P.R., \& Gale, M.D. (1988). Location of $\beta$-amylase sequence in wheat and its relatives. Theoretical and Applied Genetics, 75, 286-290. http://dx.doi.org/10.1007/BF00303966

Singh, R.P., \& Rajaram, S. (1993). Genetics of adult plant resistance to stripe rust in ten spring bread wheats. Euphytica, 72, 1-7. http://dx.doi.org/10.1007/BF00023766

Singh, R.P., Mujeeb-Kazi, A., \& Huerta-Espino, J. (1998). Lr46: A gene conferring slow-rusting resistance to leaf rust in wheat. Phytopathology, 88, 890-894. http://dx.doi.org/10.1094/PHYTO.1998.88.9.890

Singh, R.P., Huerta-Espino, J., \& William, H.M. (2005). Genetics and breeding for durable resistance to leaf and stripe rusts in wheat. Turkish Journal of Agriculture and Forestry, 29, 121-127.

Somers, D.J., Isaac, P., \& Edwards, K. (2004). A high-density microsatellite consensus map for bread wheat (Triticum aestivum L.). Theoretical and Applied Genetics, 109, 1105-1114. http://dx.doi.org/10.1007/s00122-004-1740-7

Song, Q.J., Fickus, E.W., \& Cregan, P.B. (2002). Characterization of trinucleotide SSR motifs in wheat. Theoretical and Applied Genetics, 104, 286-293. http://dx.doi.org/10.1007/s001220100698

Sourdille, P., Singh, S., Cadalen, T., Brown-Guedira, G.L., Gay, G., Qi, L., Gill, B.S., Dufour, P., Murigneux, A., \& Bernard, M. (2004). Microsatellite-based deletion bin system for the establishment of genetic-physical map relationships in wheat (Triticum aestivum L.). Functional \& Integrative Genomics, 4, 12-25. http://dx.doi.org/10.1007/s10142-004-0106-1

Wan, A.M., Chen, X.M., \& He, Z.H. (2007). Wheat stripe rust in China. Australian Journal of Agricultural Research, 58, 605-619. http://dx.doi.org/10.1071/AR06142

Wan, A.M., Zhao, Z.H., Chen, X.M., He, Z.H., Jin, S.L., Jia, Q.Z., Yao, G., Yang, J.X., Wang, B.T., Li, G.B., Bi, Y.Q., \& Yuan, Z.Y. (2004). Wheat stripe rust epidemic and virulence of Puccinia striiformis $\mathrm{f}$. sp. tritici in China in 2002. Plant Disease, 88, 896-904. http://dx.doi.org/10.1094/PDIS.2004.88.8.896

Wang, J.K., Li, H.H., Zhang, L.Y., Li, C.H., \& Meng, L. (2010). QTL IciMapping v3.0. http://www.isbreeding.net

William, H.M., Singh, R.P., Huerta-Espino, J., Ortiz-Islas, S., \& Hoisington, D. (2003). Molecular marker mapping of leaf rust resistance gene $\mathrm{Lr} 46$ and its association with stripe rust resistance gene $\mathrm{Yr} 29$ in wheat. Phytopathology, 93, 153-159. http://dx.doi.org/10.1094/PHYTO.2003.93.2.153

William, H.M., Singh, R.P., Huerta-Espino, J., Palacios, G., \& Suenaga, K. (2006). Characterization of genetic loci conferring adult plant resistance to leaf rust and stripe rust in spring wheat. Genome, 49, 977-990.

Yang, R.C. (2007). Mixed-model analysis of crossover genotype-environment interactions. Crop Science, 47, 1051-1062. http://dx.doi.org/10.2135/cropsci2006.09.0611

Zhu, H.Z., Wang, Z.W., Wu, L., Singh, R.P., Huerta-Espino, J., He, Z.H., Hu, J., Chen, F., \& Xia, X.C. (2010). QTL mapping for adult-plant resistance to stripe rust in wheat cultivar Chuanmai 107. Scientia Agricultura Sinica, 43, 706-712(in Chinese with English abstract). http://dx.doi.org/10.3864/j.issn.0578-1752.2010.04.006 
Table 1. Analysis of variance of maximum disease severities (MDS) for stripe rust on RILs derived from crosses cv. Chuanyu 16/ cv. Chuanyu 12 and cv. Chuanmai 32/cv. Chuanyu 16

\begin{tabular}{|c|c|c|c|c|c|}
\hline Population & Source of variation & $d f$ & Sum of squares & Mean square & $F$ value \\
\hline \multirow{6}{*}{$\begin{array}{l}\text { cv. Chuanyu 16/ } \\
\text { cv. Chuanyu } 12\end{array}$} & Block & 6 & 72640.5 & 12106.8 & $71.5^{* *}$ \\
\hline & Environments & 3 & 152285.8 & 50761.9 & $299.7 * *$ \\
\hline & Lines & 139 & 863262.8 & 6210.5 & $36.7 * *$ \\
\hline & Line $\times$ environment & 417 & 238465.0 & 571.9 & $3.4 * *$ \\
\hline & Error & 833 & 141114.1 & 169.4 & \\
\hline & Corrected total & 1398 & 1473479.0 & & \\
\hline \multirow{6}{*}{$\begin{array}{l}\text { cv. Chuanmai 32/ } \\
\text { cv. Chuanyu } 16\end{array}$} & Block & 6 & 60291.5 & 10048.6 & $64.7 * *$ \\
\hline & Environments & 3 & 98569.8 & 32856.6 & $211.6^{* *}$ \\
\hline & Lines & 139 & 683689.1 & 4918.6 & $31.7^{* *}$ \\
\hline & Line $\times$ environment & 417 & 150683.0 & 361.4 & $2.3 * *$ \\
\hline & Error & 834 & 129479.7 & 155.3 & \\
\hline & Corrected total & 1399 & 1120971.0 & & \\
\hline
\end{tabular}

** Significance at $P<0.0001$

Table 2. QTLs for APR To stripe rust detected in two RIL populations derived from crosses cv. Chuanyu 16/ cv. Chuanyu 12 and cv. Chuanmai 32/ cv. Chuanyu 16

\begin{tabular}{|c|c|c|c|c|c|c|c|}
\hline Population & $\begin{array}{l}\text { Location and } \\
\text { year }\end{array}$ & $\mathrm{QTL}^{\mathrm{a}}$ & Marker interval & $\mathrm{AE}^{\mathrm{b}}$ & $\mathrm{LOD}^{\mathrm{c}}$ & $\begin{array}{l}\text { PVE } \\
(\%)^{d}\end{array}$ & $\begin{array}{l}\text { Total } \\
\text { PVE }^{\mathrm{e}} \\
(\%)\end{array}$ \\
\hline \multirow{14}{*}{$\begin{array}{l}\text { cv. Chuanyu 16/ } \\
\text { cv. Chuanyu } 12\end{array}$} & \multirow[t]{2}{*}{ Chengdu 2005} & QYr.caas-1BL.2 & Xgwm818-Xgwm259 & 6 & 2.1 & 5.5 & \multirow[t]{2}{*}{23.6} \\
\hline & & QYr.caas- $2 A S$ & Xcfd036- Xwmc598 & 10.8 & 6.1 & 18.1 & \\
\hline & \multirow[t]{2}{*}{ Chengdu 2006} & QYr.caas-1BL.1 & Xbarc61-Xwmc134 & 6.2 & 2.3 & 6.0 & \multirow[t]{2}{*}{43.3} \\
\hline & & QYr.caas- $2 A S$ & Xcfd036- Хwmc598 & 15.4 & 13.8 & 37.3 & \\
\hline & Yaan 2006 & QYr.caas $-2 A S$ & Xcfd036- Хwmc598 & 22.4 & 16.1 & 43.0 & 43.0 \\
\hline & \multirow[t]{2}{*}{ Chengdu 2007} & QYr.caas- 1 BL.2 & Xgwm818-Xgwm259 & 6.3 & 2.2 & 4.5 & \multirow[t]{2}{*}{32.3} \\
\hline & & QYr.caas $-2 A S$ & Xcfd036- Xwmc598 & 15.4 & 10.8 & 27.8 & \\
\hline & \multirow[t]{3}{*}{ Yaan 2007} & QYr.caas-1BL.1 & Xbarc61-Xwmc134 & 13.3 & 3.3 & 12.8 & \multirow[t]{3}{*}{32.8} \\
\hline & & QYr.caas-1BL.2 & Xgwm818-Xgwm259 & 8.5 & 2.1 & 5.1 & \\
\hline & & QYr.caas- $2 A S$ & Xcfd036- Xwmc598 & 14.3 & 5.4 & 14.9 & \\
\hline & Yaan 2008 & QYr.caas $-2 A S$ & Xcfd036-Xwmc598 & 15.8 & 8.6 & 24.7 & 24.7 \\
\hline & \multirow[t]{3}{*}{ Average } & QYr.caas-1BL.1 & Xbarc61-Xwmc134 & 8.6 & 4.0 & 11.2 & \multirow[t]{3}{*}{49.2} \\
\hline & & QYr.caas-1BL.2 & Xgwm818-Xgwm259 & 6.3 & 3.1 & 5.8 & \\
\hline & & QYr.caas- $2 A S$ & Xcfd036- Xwmc598 & 14.6 & 14.4 & 32.2 & \\
\hline \multirow[t]{7}{*}{$\begin{array}{l}\text { cv. Chuanmai 32/ } \\
\text { cv. Chuanyu } 16\end{array}$} & Chengdu 2005 & QYr.caas- $2 A S$ & $\begin{array}{l}\text { Xwmc382- } \\
\text { VENTRIUP-LN2 }\end{array}$ & -14.1 & 13.0 & 35.5 & 35.5 \\
\hline & Chengdu 2006 & QYr.caas $-2 A S$ & $\begin{array}{l}\text { Xwmc382- } \\
\text { VENTRIUP-LN2 }\end{array}$ & -16.2 & 25.5 & 57.2 & 57.2 \\
\hline & Yaan 2006 & QYr.caas $-2 A S$ & $\begin{array}{l}\text { Xwmc382- } \\
\text { VENTRIUP-LN2 }\end{array}$ & -19.3 & 18.1 & 45.9 & 45.9 \\
\hline & Chengdu 2007 & QYr.caas $-2 A S$ & $\begin{array}{l}\text { Xwmc382 } \\
\text { VENTRIUP-LN2 }\end{array}$ & -18.8 & 20.0 & 49.8 & 49.8 \\
\hline & Yaan 2007 & QYr.caas- $2 A S$ & $\begin{array}{l}\text { Xwmc382- } \\
\text { VENTRIUP-LN2 }\end{array}$ & -11.2 & 10.0 & 29.0 & 29.0 \\
\hline & Yaan 2008 & QYr.caas $-2 A S$ & $\begin{array}{l}\text { Xwmc382- } \\
\text { VENTRIUP-LN2 }\end{array}$ & -11.1 & 10.0 & 27.9 & 27.9 \\
\hline & Average & QYr.caas- $2 A S$ & $\begin{array}{l}\text { Xwmc382- } \\
\text { VENTRIUP-LN2 }\end{array}$ & -15.1 & 24.9 & 56.9 & 56.9 \\
\hline
\end{tabular}

\footnotetext{
${ }^{a}$ QTL that extend across single one-log support confidence intervals were assigned the same symbol.

${ }^{\mathrm{b}} \mathrm{AE}$ indicated additive effect of resistance allele; positive value in the first cross and negative values in the second indicate that the QTL came from Chuanyu 16.

${ }^{c}$ LOD indicated Logarithm of odds score.

${ }^{\mathrm{d}}$ PVE indicated the phenotypic variance explained by individual QTL.

${ }^{\mathrm{e}}$ Total PVE indicated the phenotypic variance explained by all QTLs in one environment.
} 
Table 3. Summary of digenic epistatic QTLs in a RIL population derived from Chuanyu 16/Chuanyu 12 across environments

\begin{tabular}{|c|c|c|c|}
\hline Location and year & $\mathrm{QTL}_{1} \times \mathrm{QTL}_{2}$ & $\mathrm{LOD}^{\mathrm{a}}$ & $\operatorname{PVE}(\%)^{\mathrm{b}}$ \\
\hline Chengdu 2005 & QYr.caas- 1 BL. $2 \times Q Y$ Yr.caas $-2 A S$ & 4.3 & 10.4 \\
\hline Chengdu 2006 & QYr.caas -1 BL. $2 \times Q Y$ Yr.caas $-2 A S$ & 3.0 & 5.6 \\
\hline Yaan 2006 & QYr.caas- 1 BL. $2 \times Q Y$ r.caas $-2 A S$ & 2.3 & 4.3 \\
\hline Chengdu 2007 & QYr.caas- 1 BL. $2 \times Q Y$ Yr.caas $-2 A S$ & 5.9 & 10.0 \\
\hline Yaan 2007 & QYr.caas $-1 B L .2 \times Q Y$ r.caas $-2 A S$ & 2.3 & 5.7 \\
\hline Yaan 2008 & QYr.caas $-1 B L .2 \times Q Y$ r.caas $-2 A S$ & 1.9 & 4.4 \\
\hline Average & QYr.caas $-1 B L .2 \times Q Y$ Yr.caas $-2 A S$ & 4.1 & 6.7 \\
\hline
\end{tabular}

${ }^{\text {a }}$ LOD indicated Logarithm of odds score.

${ }^{\mathrm{b}}$ PVE indicated the phenotypic variance explained by digenic epistatic QTL.

Table 4. Four QTLs for stripe rust response on chromosome 1BL that could be $\operatorname{Yr} 29$

\begin{tabular}{|l|l|l|l|l|}
\hline QTL & Carrier & Interval & PVE (\%) & Reference \\
\hline QYr.cimmyt-1BL & Pavon 76 & Xgwm140 - Xgwm259 & $33-40$ & William et al. 2006 \\
\hline QYr.csiro-1BL & Attila & LTN-XP55/M55 & & Rosewarne et al. 2008 \\
\hline QPst.jic-1B & Guardian & Xgwm818-Xgwm259 & $22-45$ & Melichar et al. 2008 \\
\hline QYr.saas-1BL & Chuanmai 107 & Xcwem32-Xgwm818 & 27.4 & Zhu et al. 2010 \\
\hline
\end{tabular}

${ }^{a}$ PVE indicated the phenotypic variance explained by individual QTL. 

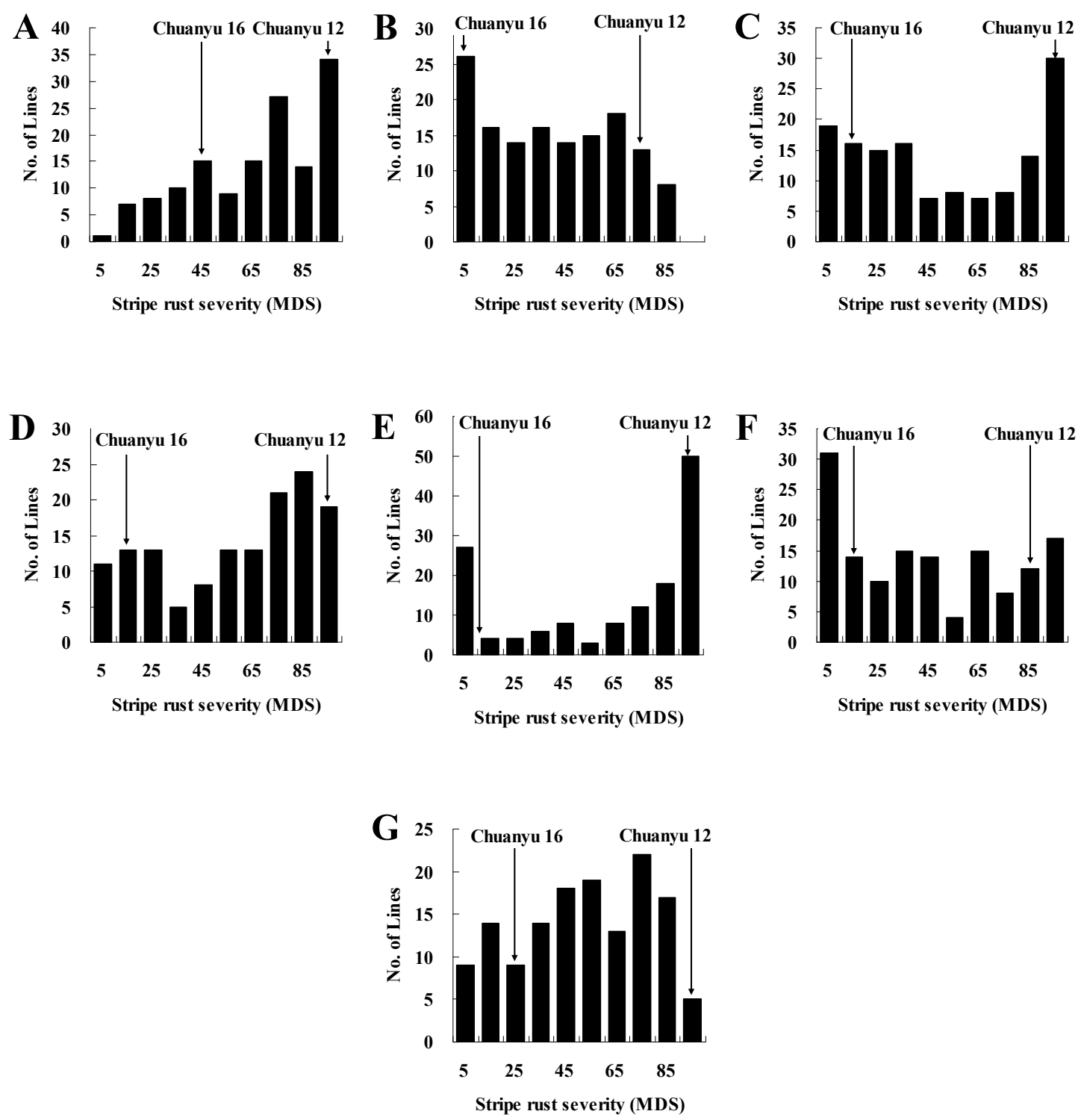

Figure 1. A to G, Frequency distributions of stripe rust maximum disease severities (MDS) in a RIL population derived from cv. Chuanyu 16/cv. Chuanyu 12. A, Chengdu 2005; B, Chengdu 2006; C, Yaan 2006; D, Chengdu 2007; E, Yaan 2007; F, Yaan 2008; G, Averaged MDS across six environments. Mean disease severities for the parents, cv. Chuanyu 16 and cv. Chuanyu 12, are indicated by arrows

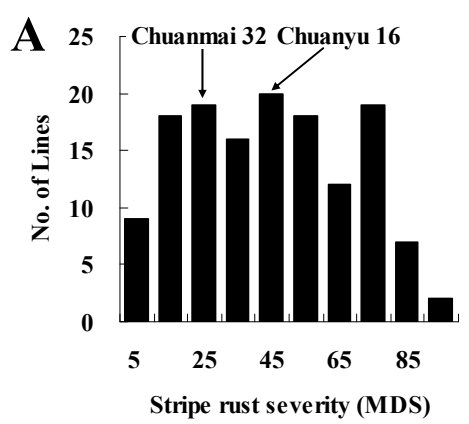

Stripe rust severity (MDS)
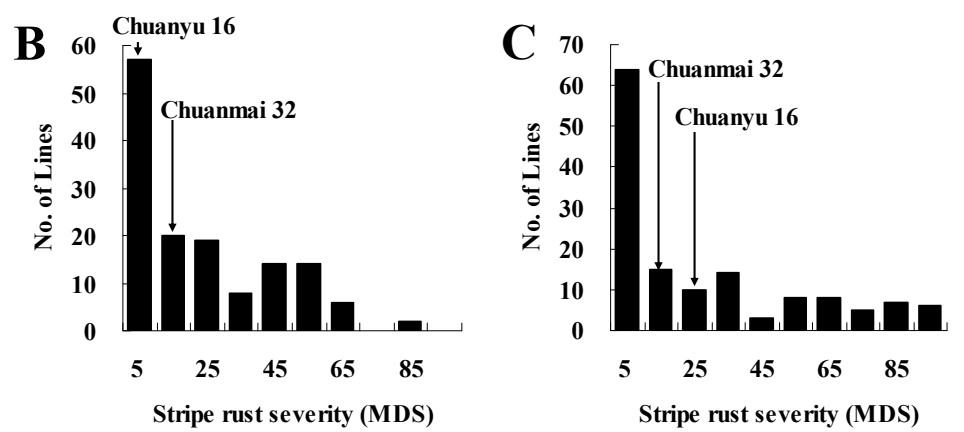

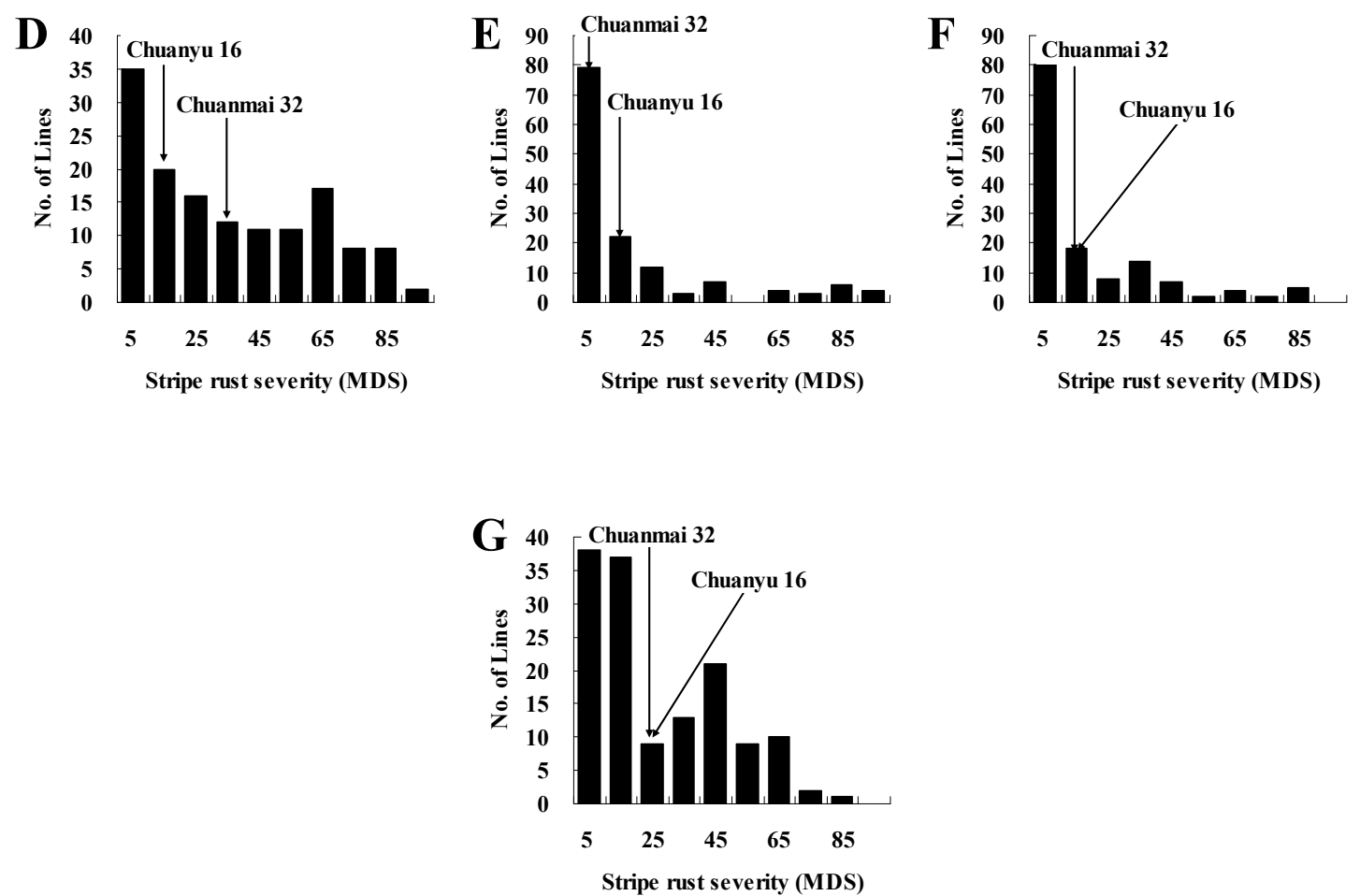

Figure 2. A to G, Frequency distributions of stripe rust maximum disease severities (MDS) in a RIL population derived from cv. Chuanmai 32/cv. Chuanyu 16. A, Chengdu 2005; B, Chengdu 2006; C, Yaan 2006; D, Chengdu 2007; E, Yaan 2007; F, Yaan 2008; G, Averaged MDS across six environments. Mean disease severities for the parents, cv. Chuanmai 32 and cv. Chuanyu 16, are indicated by arrows
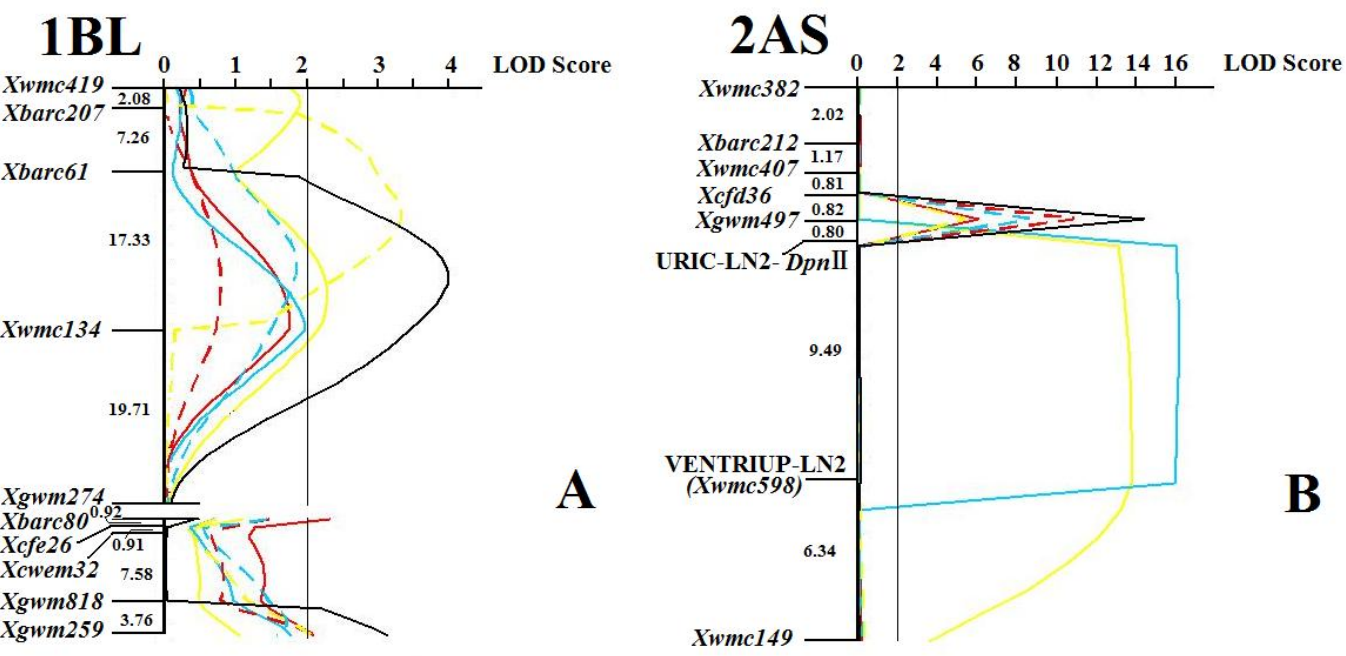


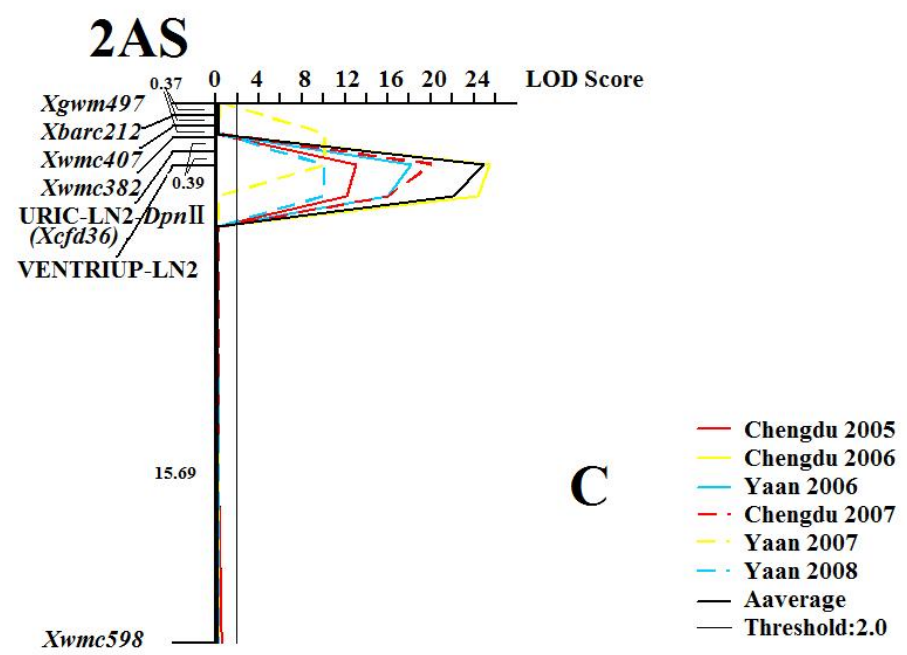

Figure 3. Likelihood plots of QTLs for APR to stripe rust on chromosomes 1BL (A), 2AS (B) identified by inclusive composite interval mapping in the population cv. Chuanyu 16/cv. Chuanyu 12; 2AS (C) identified by inclusive composite interval mapping in the population cv. Chuanmai 32/cv. Chuanyu 16. The LOD threshold for significance is 2.0. Positions (in $\mathrm{cM}$ ) of the molecular markers along chromosomes are shown on the vertical axis; numbers between marker names are genetic distances between SSR loci. Short arms are toward the top. 